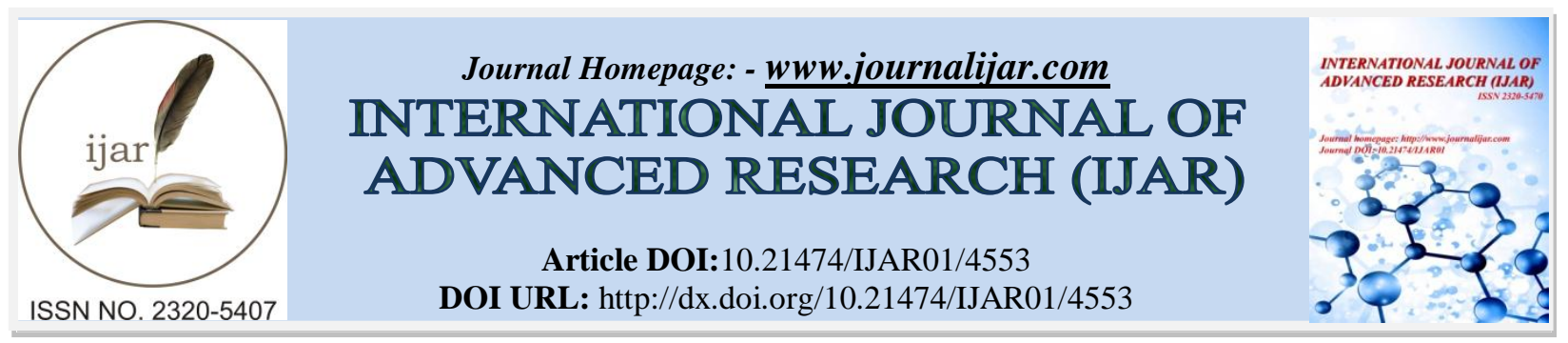

RESEARCH ARTICLE

\title{
A POTENTIAL BENEFICIAL EFFECT OF ULTRAVIOLET PHOTOTHERAPY ON RELAPSING REMITTING MULTIPLE SCLEROSIS.
}

\author{
Shereen M. Fathi ${ }^{1}$, Asmaa M. Ebraheim ${ }^{1}$, "Dalia M. Labib ${ }^{1}$, Neveen M ElFayoumy ${ }^{2}$, Amal S Ashour ${ }^{1}$, \\ AbdAlazim M Reda ${ }^{1}$, Wael Ezzat ${ }^{1}$ and Fatma Mohamed Taha ${ }^{3}$. \\ 1. Neurology Department, Faculty of Medicine, Cairo University, Egypt. \\ 2. Neurophysiology unit, Faculty of Medicine, Cairo University, Egypt. \\ 3. Medical biochemistry and molecular biology Department, Faculty of Medicine, Cairo University, Egypt.
}

\section{Manuscript Info}

\section{Manuscript History}

Received: 21 April 2017

Final Accepted: 23 May 2017

Published: June 2017

Key words:-

Multiple sclerosis, Vitamin D,

Ultraviolet Therapy.

\section{Abstract}

Background: Multiple sclerosis (MS) is an autoimmune disease of the central nervous system. Ultraviolet radiation (UVR) exposure has an influence on immune system regulation.

Aim of work: The current study was designed to elicit the effect of ultraviolet B (UVB) phototherapy on the clinical and neurophysiological outcomes in a group of relapsing remitting MS patients. A secondary aim was to clarify if vitamin D had a contributing effect in any expected change in the studied parameters.

Patients and methods: In this prospective study, 20 patients with clinically definite relapsing remitting multiple sclerosis underwent UVB phototherapy sessions for one month. Patients were initially assessed clinically and electrophysiologically before and after phototherapy, using the Expanded Disability Status Scale (EDSS), the International Prostate Symptom Score (IPSS), the Brief Ataxia Rating Scale (BARS), Modified Ashworth Scale for grading Spasticity (MAS), visual evoked potential (VEP) and H-reflex. Moreover, serum 25 hydroxy-cholecalcifirol $(25(\mathrm{OH}) \mathrm{D})$ levels (pre, post -1 month and post- 3 months UVR therapy sessions) were detected.

Results: After UVB phototherapy, the percentage of patients with sensory symptoms, unlike patients with sphincteric complains, reduced significantly $(\mathrm{P}=0.021)$. A statistically significant improvement was elicited in EDSS $(\mathrm{P}=0.01)$, IPSS $(\mathrm{P}=0.02)$ and BARS ( $\mathrm{P}=0.02)$.Furthermore, the latencies of right sided VEP $(\mathrm{P}=0.019)$ and H-reflex $(\mathrm{P}=0.001)$ were significantly reduced. Parallel to the documented improvement, follow up serum 25(OH)D levels were significantly increased. Also, a statistically significant inverse correlation between IPSS score (after one month) and the amount of elevation in $25(\mathrm{OH}) \mathrm{D}$ was found $(\mathrm{P}=0.028)$.

Conclusion: There was a beneficial effect of UVB phototherapy on clinical and neurophysiological features of MS patients, in which vitamin D improvement had a considerable contribution. 


\section{Introduction:-}

Accumulating data have implicated that environmental factors such as ultraviolet radiation (UVR) exposure has an influence on immune system regulation (Hart et al., 2002; Schwarzand Schwarz, 2010). Multiple sclerosis (MS) is an autoimmune disease of the central nervous system. Among its epidemiological features are the presence of a positive latitude gradient for disease prevalence (van der Mei et al., 2011) and a seasonal variation in disease expression (Staples et al., 2010). Sun exposure during all phases of life has a beneficial effect on patients with multiple sclerosis (van der Mei et al., 2011).Immunomodulation in response to UVR may involve the formation of vitamin D in the irradiated skin, in addition to other non-vitamin D dependent pathways (Schwarzand Schwarz, 2010; Sreevidya et al., 2010). As the main evidence for a beneficial effect of ultraviolet radiation and vitamin D for MS comes from observational and animal studies, the current study was designed to elicit the effect of ultraviolet B (UVB) phototherapy on the clinical and neurophysiological outcomes in a group of relapsing remitting MS patients. Circulating 25 hydroxy-cholecalcifirol level $(25(\mathrm{OH}) \mathrm{D})$, being largely UVB-derived via conversion of steroid precursors in the skin (Holick, 1994), was evaluated to clarify its contributing effect in any expected change in the studied parameters.

\section{Aim of work:-}

The current study was designed to elicit the effect of ultraviolet B (UVB) phototherapy on the clinical and neurophysiological outcomes in a group of relapsing remitting MS patients. A secondary aim was to clarify if vitamin D had a contributing effect in any expected change in the studied parameters.

\section{Patients and Method:-}

This was a prospective study conducted on 20 patients with clinically definite relapsing remitting multiple sclerosis diagnosed according to the McDonald's criteria and its revision 2010 (McDonald et al., 2001; Polman et al., 2011) with age ,from 20 to 46years. Patients were collected from neurology outpatient clinic and neurology department in Kasr AL-Ainy Hospital during the period from December 2012 to April 2014. The patients underwent UVR therapy sessions during their remission period with history of methylprednisolone therapy during previous exacerbations.

Patients with medical conditions that may affect the levels of vitamin D or who are contraindicated for UVR therapy were excluded. These include patients; taking vitamin D, on immunosuppressive or interferon, photosensitivity, diabetic, renal failure or liver disease, pregnant patients, pacemaker or heart failure, hyperthyroidism, traumatic brain or spinal cord injury, HIV, exposed to radiation, Patients with open wound/scar tissue on neck/back, malignancy and patient not completed his UVR therapy sessions.

\section{All patients were subjected to the following:-}

1.Full history taking, complete general and neurological examinations. Evaluation of disability using the expanded disability status scale (EDSS) (Kurtzke, 1983), The International Prostate Symptom Score (I-PSS), The Brief Ataxia Rating Scale (BARS) (Schmahmann et al., 2009) and Modified Ashworth Scale for grading Spasticity (MAS) (Bohannon and Smith, 1997).

2.Serum vitamin D level (pre, post 1 month and post 3 months UVR therapy sessions) was measured using Human 25 hydroxy-cholecalcifirol $(25(\mathrm{OH}) \mathrm{D}$; all in the same laboratory. Blood samples were examined using a doubleantibody sandwich enzyme-linked immunosorbent assay (ELISA) to assay the level of Human 25-Dihydroxy vitamin D(25-OH-D) in samples (Sunred Biological Technology, UK). The 25-OH-D is added to monoclonal antibody Enzyme well which is pre-coated with Human 25-OH-D monoclonal antibody, after incubation; 25-OH-D antibodies labeled with biotin are added, and combined with Streptavidin-HRP to form immune complex; then carry out incubation and washing are done to remove the uncombined enzyme. The intensity of color and the concentration of the Human Substance 25-Dihydroxy vitamin D (25-OH-D) of sample are positively correlated.

\section{Neurophysiological Assessment:-}

a- Visual evoked potential (VEP): VEP was performed at the Clinical Neurophysiology department, Kasr Alainy Hospital. VEP was performed (pre/post) UVR therapy. The active electrode was placed $5 \mathrm{~cm}$ above the inion and the reference electrode was placed over the forehead $(\mathrm{Fz})$, according to the 10-20 international system of electrode application and the ground one was placed over the ear tragus. The stimulus used was an alternating checkerboard pattern. We stimulated each eye separately with a check size of 320 with a stimulus rate at 1 hertz $(\mathrm{Hz})$. The patient was seated at a distance of 1 meter from the pattern stimulator and was asked to fix on a small spot placed in the center of the monitor. 
b- b- Hoffmann reflex (H-reflex): was performed at the Clinical Neurophysiology department, Kasr Alainy Hospital. H-reflex was performed (pre \& post) UVR therapy.

$\mathrm{H}$ reflexes are obtained readily with percutaneous stimulation and surface recording techniques. Stimulus pulses of long duration $(1 \mathrm{msec})$ are used to activate the large sensory fibers preferentially. Stimulus frequency should be 0.2 $\mathrm{Hz}$ or less to allow recovery of post-activation depression of the $\mathrm{H}$ reflex from a prior stimulus.

For calf $\mathrm{H}$ reflex, the posterior tibial nerve is stimulated in the popliteal fossa, recording from the soleus muscle, while $\mathrm{H}$ reflex in the forearm is recorded readily from the flexor carpi radialis muscle and the median nerve is stimulated percutaneously in the cubital fossa.

\section{UltraViolet Radiation Therapy:-}

The objective of Vitamin-D phototherapy is to use the UVB light generated by the device (at Egyptian National Institute for Laser science Cairo University)to create the natural biological reaction in the skin that results in the formation of Vitamin-D. The amount of Vitamin-D produced in the body is directly related to the number of UVB photons (light particles) that penetrate the skin to the biologically active skin layers known as the stratum-spinosum and stratum-basale.

Each Patient underwent broad band UVB phototherapy ( 3 sessions/week) for a total of 12 sessions. We used full body devices such as the 6-foot high SolRx E-Series or 1000-Series to provide the largest treatment area and therefore require the fewest number of patient body positions for full skin area coverage (Solarc/SolRx Vitamin-D User's Manual, 2012). It had shown that a single dose of whole-body sub-erythemal UVB (characterized by mild pinkness of the skin, just below the state of mild sunburn) can create the equivalent of 10,000 to 20,000 IU of oral Vitamin-D. However, this is the maximum dose that ever ought to be taken as it exposes the patient to greater risk of burning. It is instead likely safer to take more frequent smaller doses.

The study was approved by Cairo University Hospitals Research Ethics Committee and written informed consents were obtained from patients.

\section{Statistical Analysis:-}

Data were statistically described in terms of mean \pm standard deviation $( \pm$ SD), median and range for quantitative variables, and frequencies (number of cases) and percentages for qualitative variables. Comparison was done using chi square test for quantitative variables. Multiple comparisons were done using post Hoc test for normally distributed quantitative variables and parametrical. Mann-Whitney test for not normally distributed quantitative variables. The Chi-squared test for association is the most common method used for the measurement of the strength of association between two qualitative variables. P values less than 0.05 was considered statistically significant. All statistical calculations were done using computer programs SPSS (Statistical Package for the Social Science; SPSS Inc., Chicago, IL, USA) version 12 for Microsoft Windows.

\section{Results:-}

\section{Demographic and clinical profile of patients:-}

The age of the patients ranged from 20 - 46 years $(31.15 \pm 6.79$, mean \pm SD). Female patients $(n=11)$ represented a relatively larger proportion (55\%) compared to the male patients $(n=9)(45 \%)$. The duration of illness ranged from 18 months to 10 years. The Number of attacks prior to participation ranged from $2-9$ attacks $(4.75 \pm 1.77$, mean \pm SD).

Effects of one month UVB phototherapy on neurological systems involved are shown in Table (1).

Comparison between different clinical scales pre and post one month UVB phototherapy (table 2)

Comparison between the measured neurophysiologic parameters pre and post one month UVB phototherapy (table3)

An elevation was detected in serum25 hydroxy-cholecalcifirol25(OH) D level one month after the initiation of UVB phototherapy. Vitamin D mean levels continued to increase for the next 2 months despite the discontinuation of therapy, these elevations were statistically significant, table 4 .

Four patients (20\%) had their 25(OH)D levels decreased or unchanged following UVB phototherapy sessions. Three of them were featured by lack of improvement in both clinical and neurophysiological parameters and experienced relapses after they completed one month of UVB phototherapy. 
Only statistically significant inverse correlation between IPSS score after one month of UVB phototherapy and $25(\mathrm{OH}) \mathrm{D}_{\delta}(\mathrm{P}=0.028)$ was found that means he more the amount of increase of 25 hydroxy-cholecalcifirol, the more the improvement in the IPSS score, table 5.

There were no statistically significant correlations between the amount of increase of serum 25 hydroxycholecalcifirol level $\left[25(\mathrm{OH}) \mathrm{D}_{\delta}\right]$ and the neurophysiological tests after one month of UVB phototherapy, table 6.

Table 1:- Effect of UVB phototherapy (for one month) on different neurological deficits of the patients.

\begin{tabular}{|c|c|c|c|}
\hline \multirow{2}{*}{ Patients prior to therapy (n=20) } & \multicolumn{2}{|c|}{ Effect of UVB phototherapy } & \multirow{2}{*}{ p-value } \\
\cline { 2 - 3 } & $\begin{array}{c}\text { Improved } \\
\text { No }(\boldsymbol{\%})\end{array}$ & Unimproved No (\%) & 0.12 \\
\hline Cranial nerves involvement (n=9) & $4(44.4)$ & $5(55.6)$ & 0.50 \\
\hline Motor deficit (n=15) & $2(13.3)$ & $13(86.7)$ & 0.50 \\
\hline Coordination deficit (n=11) & $2(18.2)$ & $9(81.8)$ & $0.021^{*}$ \\
\hline Sensory deficit (n=12) & $10(83.3)$ & $2(16.7)$ & $0.005^{*}$ \\
\hline Sphincteric troubles $(\mathbf{n}=\mathbf{1 2})$ & $4(33.3)$ & $8(66.7)$ & \\
\hline
\end{tabular}

Data are presented as number and (\%); UVB, ultraviolet rays B band; *significant.

Table 2:- Comparison between the median scores of different clinical scales pre and post one month UVB phototherapy

\begin{tabular}{|c|c|c|c|}
\hline Clinical scale & Pre-UVB phototherapy & Post-UVB phototherapy & P-value \\
\hline EDSS & $3.5(1.5-6)$ & $2.75(1.5-5.5)$ & $0.01^{*}$ \\
\hline IPSS & $2(0-7)$ & $0(0-6)$ & $0.02^{*}$ \\
\hline MAS & $0(0-3)$ & $0(0-3)$ & 0.31 \\
\hline BARS & $3(0-15)$ & $1(0-13)$ & $0.02^{*}$ \\
\hline
\end{tabular}

Data are presented as median and (range). UVB, ultraviolet rays B band; EDSS, Expanded Disability Status Scale; IPSS, The International Prostate Symptom Score ;MAS, Modified Ashworth Scale; BARS, The Brief Ataxia Rating Scale; *significant.

Table 3:- Comparison between the measured neurophysiologic parameters pre and post one month UVB phototherapy

\begin{tabular}{|c|c|c|c|}
\hline Neurophysiologic test & Pre-UVB phototherapy & Post-UVB phototherapy & P-value \\
\hline Rt VEP amplitude, $\boldsymbol{\mu V}$. & $7.30 \pm 3.52$ & $7.60 \pm 3.51$ & 0.05 \\
\hline Lt VEP amplitude, $\boldsymbol{\mu V}$. & $7.60 \pm 4.26$ & $8.00 \pm 4.37$ & 0.07 \\
\hline Rt VEP latency, msec. & $121.20 \pm 15.67$ & $119.89 \pm 15.51$ & $0.019 *$ \\
\hline Lt VEP latency, msec. & $123.86 \pm 20.84$ & $121.96 \pm 18.05$ & 0.10 \\
\hline $\begin{array}{c}\text { UL H-reflex latency, } \\
\text { msec. }\end{array}$ & $24.13 \pm 9.75$ & $23.59 \pm 9.74$ & $0.001^{*}$ \\
\hline $\begin{array}{c}\text { LL H-reflex latency, } \\
\text { msec. }\end{array}$ & $29.96 \pm 7.17$ & $28.96 \pm 6.68$ & $0.001^{*}$ \\
\hline
\end{tabular}

Data are presented as mean \pm SD; UVB, ultraviolet rays B band; VEP, visual evoked potential; Rt, right; Lt, left; UL, upper limb; LL, lower limb; *significant.

Table 4:- Comparison between serum levels of 25 hydroxy-cholecalcifirol in relation to initiation of one month UVB phototherapy.

\begin{tabular}{|c|c|c|}
\hline 25(OH)D, ng/mL & Level & P-value \\
\hline Pre-therapy & $<0.001^{*}$ \\
\hline After 1 month & $31.05 \pm 7.45(21.5-60)$ & $0.004^{*}$ \\
\cline { 2 - 2 } $\begin{array}{c}\text { Pre- therapy } \\
\text { After 3 months }\end{array}$ & $34.66 \pm 5.86(28.5-55)$ & $0.003^{*}$ \\
\cline { 2 - 2 } $\begin{array}{c}\text { After 1 month } \\
\text { After 3 month }\end{array}$ & $31.05 \pm 7.45(21.5-60)$ & \multirow{2}{*}{0} \\
\cline { 2 - 2 } & $37.87 \pm 9.81(28.4-77)$ & $34.66 \pm 5.86(28.5-55)$ \\
\end{tabular}

Data are presented as mean \pm SD and (range); UVB, ultraviolet rays B band; 25(OH)D, 25 hydroxy-cholecalcifirol; *significant. 
Table 5:- Correlation between the amount of increase of serum 25 hydroxy-cholecalcifirol level $\left[25(\mathrm{OH}) \mathrm{D}_{\delta}\right]$ and the scores of different clinical scales after one month of UVB phototherapy.

\begin{tabular}{|c|c|c|}
\hline $\mathbf{2 5}(\mathbf{O H}) \mathbf{D}_{\boldsymbol{\delta}}$ & $\mathbf{R}$ & p-value \\
\hline EDSS & 0.002 & 0.992 \\
\hline IPSS & -0.490 & $0.028^{*}$ \\
\hline MAS & 0.124 & 0.602 \\
\hline BARS & 0.600 & 0.802 \\
\hline
\end{tabular}

EDSS, Expanded Disability Status Scale; IPSS, The International Prostate Symptom Score ;MAS, Modified Ashworth Scale; BARS, The Brief Ataxia Rating Scale ; r, correlation coefficient; *significant.

Table 6:- Correlation between the amount of increase of serum 25 hydroxy-cholecalcifirol level $\left[25(\mathrm{OH}) \mathrm{D}_{\delta}\right]$ and the neurophysiological tests after one month of UVB phototherapy.

\begin{tabular}{|l|l|l|}
\hline $\mathbf{2 5}(\mathbf{O H}) \mathbf{D}_{\boldsymbol{\delta}}$ & $\mathbf{R}$ & p-value \\
\hline RtVEP amplitude & 0.31 & 0.18 \\
\hline Lt VEP amplitude & 0.25 & 0.28 \\
\hline RtVEP latency & -0.41 & 0.72 \\
\hline LtVEP latency & -0.33 & 0.14 \\
\hline UL H-reflex latency & -0.23 & 0.33 \\
\hline LL H-reflex latency & -0.26 & 0.27 \\
\hline
\end{tabular}

VEP, visual evoked potential; Rt, right; Lt, left; UL, upper limb; LL, lower limb; r, correlation coefficient.

\section{Discussion:-}

Although Egypt is known for receiving ample sunshine for the most part of the year, $50 \%$ of the patients in the current study had insufficient $25(\mathrm{OH})$ D levels $(<30 \mathrm{ng} / \mathrm{ml})$. In agreement with our results, several Egyptian studies have shown that vitamin D levels to be either deficient $(<20 \mathrm{ng} / \mathrm{ml})$ or insufficient in the MS patients (El-Ghoneimy et al., 2009; Esmael et al., 2016) and the controls (Zamzamet al., 2016).

In our study, there was a significant increase in $25(\mathrm{OH})$ D mean level after UVB phototherapy. Surprisingly, vitamin D mean levels continued to increase for the next 2 months despite the discontinuation of therapy.

In the present study percentage of patients with sensory complaints reduced significantly after broad band UVB phototherapy (for 3 times/week for 4 weeks). Moreover, a statistically significant improvement in the scores EDSS, IPSS and BARS were detected; on the other hand the scores of MAS did not change significantly. In agreement with our results, several studies have reported significant improvement in the EDSS of RRMS patients after either broad band UVB phototherapy as our study (Essa et al., 2016) or narrow band UVB phototherapy ( for 5times/week for 6 weeks) (Breuer et al.,2014).

As stated before, exposure to sunlight is essential for the transformation of 7-dehydrocholesterol in the skin into vitamin D3. The liver converts vitamin D3 into 25-hydroxyvitamin D3 (biologically inactive). Other tissues including, the kidney, the immune system, the intestine and the central nervous system are responsible for the step of activation into a biologically active hormone (Calcitriol) (Munger et al., 2006).

In this scenario, some authors have suggested that UVR exposure and vitamin D status may play independent roles for modulation of central nervous system demyelination. Goldberg(1974) postulated that sunlight suppresses multiple sclerosis through promoting synthesis of vitamin D. Activated vitamin D shifts CD4 T-cells and MHC class II molecules to a more anti-inflammatory profile in-vitro (Smolders et al., 2008). It could block experimental autoimmune encephalomyelitis (mouse model of MS) (Cantorna et al., 1996). Broad band UV light clearly suppressed the disease in mouse model of MS and also slightly increased serum 25OHD3. Quite opposite to the original hypotheses of Goldberg, when mice were exposed to narrow band UV light, the activity of the disease suppressed with even though unremarkable increase in serum vitamin D (Wang et al., 2013). Thus, exactly how UVR functions to influence autoimmunity is as yet unclear. Other UV-induced mechanisms are sunlight-stimulated neuronal activity that may influence antigen presentation in the brain, and UV-mediated alterations to the levels of bioactive vitamin A and melatonin (Mehta, 2010). UV irradiation of skin may modulate immunity to Epstein-Barr virus infection (van der Mei et al., 2011); such infection has been linked with the etiology of MS. In addition, UVoxidized lipids and proteins initiate signaling pathways, with release of mediators (IL-10, IL-6, prostaglandin E2, 
platelet activating factor, tumour necrosis factor and nerve growth factor)that are involved in UV-induced immunosuppression (Hart et al., 2002; Norval, 2006;Ullrich, 2005).

Unexpectedly, in the current study, four patients showed lower or unchanged 25(OH) D levels after UVB phototherapy compared to those measured before therapy sessions. Three of them experienced relapse of the disease after session completion (during the next 2 months). It may be attributed to genetic, racial and ethnic profile that may play a role in determining the individual response to ultraviolet ray exposure and hence vitamin D synthesis and MS development or outcome (Langer-Gould et al., 2015). Skin type and adiposity might be relevant factors (Woolmore et al., 2007).In addition, among MS patients living where serum vitamin D levels fluctuate seasonally, a winter drop in vitamin D usually precedes a spring surge in relapses (Munger et al., 2006). Others suggested that ongoing inflammation, as in MS, might hinder systemic increases in $25(\mathrm{OH}) \mathrm{D}$ levels even with supplementation (Bhargava et al.,2016).

Being quantifiable, neurophysiological assessment was carried out to augment the outcome of our research. In the present study, the latencies of right sided VEP and H-reflex of both upper and lower limbs were significantly reduced after phototherapy. Also, a significant positive correlation was elicited between the VEP amplitude and $25(\mathrm{OH}) \mathrm{D}_{\delta}$. The latencies of VEP and the H-reflex were negatively correlated with $25(\mathrm{OH}) \mathrm{D}_{\delta}$, however the values were not statistically significant. To date, there is paucity in studies evaluating the effect of UVB phototherapy and vitamin D on neurophysiological parameters of the MS patient. In concordance with our results Essa and his colleagues (2015) found that the broad band UVB photo therapy had a highly significant improvement on VEP latency of the right optic nerve in MS patients that was sustained at follow up. Our result was in disagreement with López-Méndeza et al. (2016) who did not observe any statistically significant relationship between vitamin D levels of MS patients and VEP results.

In the other hand, Essa and his colleagues (2016)found that nonsignificant differences in H-reflex of the right and left tibial nerves between baseline and post-phototherapy and follow-up values and this does not go with our finding. Moreover, the percentages of their patients with latencies $>32 \mathrm{~ms}$ of the right tibial nerve dropped from $50 \%$ at baseline to $16.7 \%$ after treatment and rose again to $50 \%$ at follow-up. The percentage of less than $28 \mathrm{~ms}$ latencies increased from $16.7 \%$ at baseline to $50 \%$ after treatment and dropped again to $33.3 \%$ at follow-up, indicating a transit decrease in spasticity after treatment that did not sustain during the follow-up period.

\section{Limitations:-}

Small sample size, short follow-up period and limited category of MS patients (relapsing remitting type)

\section{Conclusion:-}

There were beneficial effects of UVB phototherapy on clinical and neurophysiological features of MS, in which vitamin D improvement had a remarkable contribution. However, to evaluate the limitations and potential hazards of such therapy further extended studies are warranted.

\section{References:-}

1. Bhargava, P., Steele, S.U., Waubant, E., Revirajan, N.R., Marcus, J., Dembele, M. and et al. (2016): Multiple sclerosis patients have a diminished serologic response to vitamin D supplementation compared to healthy controls. Mult Scler., 22:753-60.

2. Bohannon, R.W., Smith, M.B. (1997): Inter rater reliability of a modified Ashworth scale of muscle spasticity. Phys Ther., 67(2):206-7

3. Breuer, J., Schwab, N., Schneider-Hohendorf, T., Marziniak, M., Mohan, H., Bhatia, U. and et al. (2014): Ultraviolet B light attenuates the systemic immune response in central nervous system autoimmunity. Ann Neurol., 75:739-58.

4. Cantorna, M.T., Hayes, C.E. and DeLuca, H.F. (1996): 1,25-Dihydroxyvitamin D3 reversibly blocks the progression of relapsing encephalomyelitis, a model of multiple sclerosis. ProcNatlAcadSci U S A., 93(15): 7861-7864.

5. El-Ghoneimy ,A.T., Gad, A.H., Samir, H., Shalaby, N.M., Ramzy, G.H., Farghaly, M. and Hegazy, M.I. (2009): Contribution of vitamin D to the pathogenesis of multiple sclerosis and its effect on bone. Egypt J Neurol .Psychiat Neurosurg., 46:209-222. 
6. Esmael, A., El-Sherifa, M. and Elazzounyb, A.A. (2016): Effects of vitamin D deficiency on the relapse, severity, and disability of multiple sclerosis. Egypt J Neurol Psychiat Neurosurg., 53(3):174-178.

7. Essa, S.A., Mostafa, Y.M., Fathi, S.M., Elhafez, H.M., Ahmed, A.F. and El Fayoumy, N.M. (2015): Could Phototherapy Reverse Visual Deficits in Patients with Relapsing Remitting Multiple Sclerosis?. JMSCR., 3(5): 5479-5494.

8. Essa, S.A., Mostafa,Y.M., Fathi, S.M., Elhafez, H.M., Ahmed, A.F. and El Fayoumy, N.M. (2016): Spasticity is modifiable through phototherapy in patients with relapsing remitting multiple sclerosis: A randomized controlled study. Kasr AL Ainy medical journal., 22(3) : 81-90.

9. Goldberg, P. (1974): Multiple sclerosis: vitamin D and calcium as environmental determinants of prevalence. Part 1: sunlight, dietary factors and epidemiology. Int J Environ Stud., 6: 19-27.

10. Hart, P. H., Townley, S. L., Grimbaldeston, M. A., Khalil, Z. and Finlay-Jones, J. J. (2002): Mast cells, neuropeptides, histamine and prostaglandins in UV-induced systemic immunosuppression. Methods., 28: 7989.

11. Holick, M. F. (1994): McCollum Award Lecture, 1994: vitamin D7— new horizons for the 21st century. Am. J Clin Nutr., 60: 619-630.

12. Kurtzke, J.F.(1983): Rating neurologic impairment in multiple sclerosis: an ExpandedDisability Status Scale (EDSS)". Neurology., 33:1444-1452.

13. Langer-Gould, A., Chen, L., Lucas, R., Xiang, A. and Barcellos, L. (2015): Hypovitaminosis D and the risk of multiple sclerosis in blacks and Hispanics. Neurology., 84(14): 2-207.

14. López-Méndeza, p., Sosa-Henríquezb, M. and Ruiz-Pérezd, Á. (2016): Relationship between vitamin D deficiency and visually evoked potentials in multiple sclerosis. archsocespoftalmol., 91 (5):217-222.

15. McDonald, W.I., Compston, A., Edan, G., Goodkin, D., Hartung, H.P., Lublin, F.D., and et al. (2001): Recommended diagnostic criteria for multiple sclerosis: guidelines from the International Panel on the diagnosis of multiple sclerosis. Ann Neurol., 50: 121-27.

16. Mehta, B. K. (2010): New hypotheses on sunlight and the geographic variability of multiple sclerosis prevalence. J Neurol Sci., 192: 5-10.

17. Munger, K.L., Levin, L.I., Hollis, B.W., Howard, N.S. and Ascherio, A. (2006): Serum 25-hydroxyvitamin D levels and risk of multiple sclerosis. JAMA., 296 :2832-2838.

18. Norval, M.(2006): The mechanisms and consequences of ultraviolet-induced immunosuppression. Prog Biophys Mol Biol., 92:108-118.

19. Polman CH, Reingold SC, Banwell B, Clanet M, Cohen JA, Filippi M and et al. (2011): Diagnostic criteria for multiple sclerosis: 2010 revisions to the McDonald criteria. Ann Neurol.; 69(2):292-302.

20. Schmahmann, J.D., MacMore, J. and Vangel, M.(2009): Ceerebeller stroke without motor deficit: clinical evidence for motor and non-motor domains within the human cerebellum. Neuroscience., 162(3):852-861.

21. Schwarz, A. and Schwarz, T. (2010): UVR-induced regulatory T cells switch antigen-presenting cells from a stimulatory to a regulatory phenotype. J Invest Dermatol.,130:1914-1921.

22. Smolders, J., Damoiseaux, J., Menheere, P. and Hupperts, R. (2008): Vitamin D as an immune modulator in multiple sclerosis, a review. J Neuroimmunol., 194(1):7-17.

23. Solarc/SolRx Vitamin-D User's Manual, 2012.

24. Sreevidya, C. S. , Fukunaga, A., Khaskhely, N.M., Masaki, T., Ono, R., Nishigori, C. and Ullrich, S.E. (2010): Agents that reverse UV-induced immune suppression and photocarcinogenesis affect DNA repair. J Invest Dermatol.,130, 1428-1437.

25. Staples, J., Ponsonby, A. L. and Lim, L. (2010): Low maternal exposure to ultraviolet radiation in pregnancy, month of birth, and risk of multiple sclerosis in offspring: longitudinal analysis. BMJ., 340. c1640.

26. Ullrich, S. E. (2005): Mechanisms underlying UV-induced immune suppression. Mutat. Res., 571: 185-205.

27. van der Mei, I. A., Simpson, S., Stankovich, J. and Taylor, B. V. (2011): Individual and joint action of environmental factors and risk of MS. Neurol Clin., 29: 233-255.

28. Wang, Y., Marling, S.J., Mcknight, S.M., Danielson, A.L., Severson, K.S. and Deluca, H.F. (2013): Suppression of experimental autoimmune encephalomyelitis by $300-315 \mathrm{~nm}$ ultraviolet light. Arch Biochem Biophys., 536:81-86.

29. Woolmore, J.A., Stone, M., Pye, E.M. and et al. (2007): Studies of associations between disability in multiple sclerosis, skin type, gender and ultraviolet radiation. Mult. Scler., 13: 369-75.

30. Zamzam, D.A., Fouad, M.M., Elaidy, D.A., Abd-Elaziz, D.M.and Abd-Elaziz, A.A. (2016): Vitamin D levels in a sample of Egyptian patients with multiple sclerosis. Egypt J Neurol Psychiat Neurosurg., 53 (2) :107-110. 